\title{
The numerical simulation of one-dimensional overland flow by Lattice
}

\section{Boltzmann Method}

\author{
Ningning LIU $\mathrm{a}^{*}$ \\ ${ }^{1}$ Water project management bureau of Shandong province, Jinan Shandong Province, China \\ aEmail liuning1123@126.com
}

\begin{abstract}
Keywords: the kinematic wave equation Lattice Boltzmann method overland flow relaxation time
\end{abstract}

Abstract: The kinematic wave equation is selected to describe one-dimensional overland flow. The Lattice Boltzmann method which different from macroscopic numerical methods is introduce to solve this partial differential equation. By comparing to analytical solution, good fitting results are got. In addition, the optimal range of relaxation time is also discussed in this paper.

\section{Introduction}

When the rainfall intensity exceed soil infiltration, water begins to accumulate on the surface and then it flows down the slope under gravity as overland flow. Overland flow is great influenced by topography, rainfall and surface roughness, so it has very complicated hydraulic characteristics. The full St Venant equations have been known to be used as mathematical equations to describe the overland flow. However these equations are very complicated and for many practical instances, such a degree of sophistication is not required. The simplified equations should be chosen whenever the physical conditions justified them. Among these, the two common models are the diffusion wave and the kinematic wave approximations ${ }^{[1,2]}$.

Numerical methods such as finite difference/element methods and method of characteristics ${ }^{[3,4]}$, etc, are the useful tools to solve these equations. However, it has been observed that the physical modeling of overland flow through numerical methods had contradictions between numerical stability and computer effort. Thus there is a distinct need for a simpler solution methodology which can be applied to overcome these contradictions. This paper presents Lattice Boltzmann method (LBM) for one-dimensional overland flow. The Lattice Boltzmann method was proposed in recent years as a numerical method for simulating fluid flows ${ }^{[5]}$. Unlike conventional numerical schemes based on discretization of macroscopic continuum equations, the lattice Boltzmann method is based on microscopic models and mesoscopic kinetic equations. It provides an indirect way to solve flow equations and it brings certain advantages over conventional numerical methods, such as parallel computation, easy handing of complex geometries, easy programming and easy simulating complex flows, such as, multiphase flows ${ }^{[6]}$, porous flows ${ }^{[7]}$, flows of suspensions ${ }^{[8]}$ and other flows with variations of boundaries. In addition, LBM has been developed to solve nonlinear partial differential equations such as, shallow water flow equation and Burgers equation ${ }^{[9,10]}$.

In this paper, the kinematic wave equation is selected to be the simplified form of St Venant equations. The Lattice Boltzmann method is used to solve this equation. It has been verified by comparing to the analytical solution which is mentioned in [11]. 


\section{Mathematical equation of overland flow}

The mathematical representation of one-dimensional overland flow is through the St Venant equations. These equations are comprised of continuity (mass balance) and momentum (energy balance) equation. These equations can be expressed as follows:

$$
\left\{\begin{array}{c}
\frac{\partial h}{\partial t}+\frac{\partial(v h)}{\partial x}=i_{e}(x, t) \\
\frac{\partial v}{\partial t}+v \frac{\partial v}{\partial x}+g \frac{\partial h}{\partial x}=g\left(S_{0}-S_{f}\right)-\frac{v}{h} i_{e}(x, t)
\end{array}\right.
$$

Where $h=$ flow depth, $v=$ flow velocity, $i_{e}(x, t)=$ net inflow rate (rainfall minus infiltration), $S_{0}=$ bed slope, $S_{f}=$ friction slope and $g=$ gravitational acceleration.

However, there are many difficulties to solve the full St Venant equations, so the kinematic wave approximation is introduced in this paper. It equates the friction slope to the bed slope and adopts the Manning roughness relationship. Thus:

$$
\left\{\begin{array}{c}
\frac{\partial h}{\partial t}+\frac{\partial q}{\partial x}=i_{e}(x, t) \\
q=\frac{1}{n} h^{\frac{5}{3}} S_{\mathrm{O}}^{\frac{1}{2}}
\end{array}\right.
$$

Where $n$ is Manning's roughness coefficient, $q$ is unit width discharge.

In this paper, the boundary condition and initial condition are:

$$
\begin{cases}h(0, t)=0 & t>0 \\ h(x, 0)=0 & 0 \leq x \leq L\end{cases}
$$

Where $L$ is the length of slope.

\section{Lattice Boltzmann model}

Lattice Boltzmann equation. Lattice Boltzmann method divides the space into uniform mesh and the particle distribution function $f_{\alpha}\left(\vec{x}, \vec{e}_{\alpha}, t\right)$ is laid on the each node. It emphasizes the average properties of particles while neglects interactions between them. Based on the physical characteristics, the lattice Boltzmann equation will be obtained as:

$$
f_{\alpha}\left(\vec{x}+\Delta t \cdot \vec{e}_{\alpha}, \vec{e}_{\alpha}, t+\Delta t\right)-f_{\alpha}\left(\vec{x}, \vec{e}_{\alpha}, t\right)=\Omega_{\alpha}\left(f_{\alpha}\left(\vec{x}, \vec{e}_{\alpha}, t\right)\right)+(\Delta t)^{2} \cdot g_{\alpha}\left(\vec{x}, \vec{e}_{\alpha}, t\right)
$$

Where $f_{\alpha}\left(\vec{x}, \vec{e}_{\alpha}, t\right)$ is the distribution function, $\Omega_{\alpha}\left(f_{\alpha}\left(\vec{x}, \vec{e}_{\alpha}, t\right)\right)$ is the collision operator which represents the rate of change, $\vec{e}_{\alpha}$ is the particle velocity, $g_{\alpha}\left(\vec{x}, \vec{e}_{\alpha}, t\right)$ is source term and in this paper is rainfall.

Usually, with the BGK approximation ${ }^{[12]}$, the collision operator $\Omega_{\alpha}\left(f_{\alpha}\left(\vec{x}, \vec{e}_{\alpha}, t\right)\right)$ is replaced by the single relaxation time $\tau$ :

$$
f_{\alpha}\left(\vec{x}+\Delta t \cdot \vec{e}_{\alpha}, \vec{e}_{\alpha}, t+\Delta t\right)-f_{\alpha}\left(\vec{x}, \vec{e}_{\alpha}, t\right)=-\frac{1}{\tau}\left(f_{\alpha}\left(\vec{x}, \vec{e}_{\alpha}, t\right)-f_{\alpha}^{e q}\left(\vec{x}, \vec{e}_{\alpha}, t\right)\right)+(\Delta t)^{2} \cdot g_{\alpha}\left(\vec{x}, \vec{e}_{\alpha}, t\right)
$$

Where $f_{\alpha}^{e q}\left(\vec{x}, \vec{e}_{\alpha}, t\right)$ is the local equilibrium distribution function.

According to the theory of the lattice Boltzmann method, it consists of two steps which are 
shown in Fig.1:(1) Streaming step, the particles move to the neighboring lattice point and replace the distribution function of it.(2) Collision step, the arriving particles at the points interact one another and change their velocity directions according to scattering rules. The two steps can be expressed as:

Collision step:

$$
\begin{aligned}
f_{\alpha}^{n e w}\left(\vec{x}, \vec{e}_{\alpha}, t\right) & =f_{\alpha}\left(\vec{x}, \vec{e}_{\alpha}, t\right)-\frac{1}{\tau}\left(f_{\alpha}\left(\vec{x}, \vec{e}_{\alpha}, t\right)-f_{\alpha}^{e q}\left(\vec{x}, \vec{e}_{\alpha}, t\right)\right) \\
& +(\Delta t)^{2} \cdot g_{\alpha}\left(\vec{x}, \vec{e}_{\alpha}, t\right)
\end{aligned}
$$

Streaming step: $\quad f_{\alpha}^{\text {new }}\left(\vec{x}+\Delta t \cdot \vec{e}_{\alpha}, \vec{e}_{\alpha}, t+\Delta t\right)=f_{\alpha}^{\text {new }}\left(\vec{x}, \vec{e}_{\alpha}, t\right)$

In the collision step, $\Omega_{\alpha}\left(f_{\alpha}\left(\vec{x}, \vec{e}_{\alpha}, t\right)\right)$ is required to satisfy conservation of total mass and total momentum at each lattice:

$$
\sum_{\alpha} \Omega_{\alpha}\left(f_{\alpha}\left(\vec{x}, \vec{e}_{\alpha}, t\right)\right)=0 \quad \sum_{\alpha} \vec{e}_{\alpha} \cdot \Omega_{\alpha}\left(f_{\alpha}\left(\vec{x}, \vec{e}_{\alpha}, t\right)\right)=0
$$
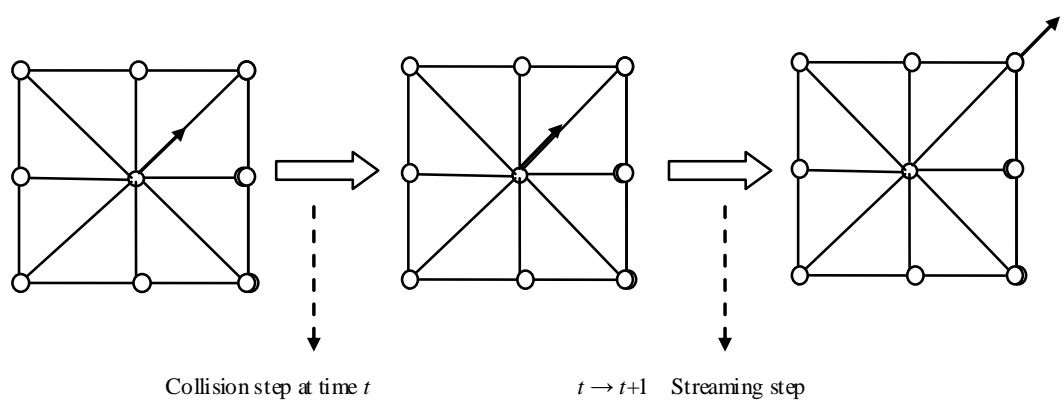

Fig 1 collision step and streaming step of Lattice Boltzmann method

Lattice Boltzmann model of one- dimensional overland flow. In this paper, a new Lattice Boltzmann model of D1Q3 three-speed model which is mentioned by J. Zhang and G. Yan ${ }^{[10]}$ is introduced. In the D1Q3 model which is shown in Fig.2, on each node, there are particles of three different velocities. The velocity vectors are: $e_{1}=-C \quad e_{0}=0 \quad e_{2}=C$.

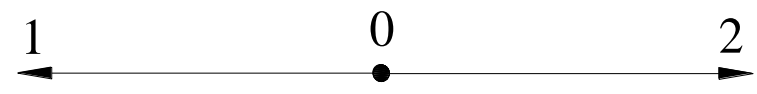

Fig.2 D1Q3 three-speed model

The new way doesn't deal with the spatial coordinate while introduce three time scales, and their differential coefficient form are:

$$
\left\{\begin{array}{c}
\frac{\partial}{\partial t}=\frac{\partial}{\partial t_{0}}+\varepsilon \frac{\partial}{\partial t_{1}}+\varepsilon^{2} \frac{\partial}{\partial t_{2}}+o\left(\varepsilon^{3}\right) \\
\frac{\partial}{\partial x}=\frac{\partial}{\partial x}+o(\varepsilon)
\end{array}\right.
$$

Where $\varepsilon$ is the Knudsen number which can be defined as is $\varepsilon=l / L, l$ is the mean free path, and $\mathrm{L}$ is the characteristic length.

Likewise, the distribution function $f_{\alpha}\left(\vec{x}, \vec{e}_{\alpha}, t\right)$ can be expanded formally about the local equilibrium distribution function $f_{\alpha}^{e q}\left(\vec{x}, \vec{e}_{\alpha}, t\right)$ :

$$
f_{\alpha}\left(\vec{x}, \vec{e}_{\alpha}, t\right)=f_{\alpha}^{e q}\left(\vec{x}, \vec{e}_{\alpha}, t\right)+\varepsilon f_{\alpha}^{n e q}\left(\vec{x}, \vec{e}_{\alpha}, t\right)
$$

Here $f_{\alpha}^{e q}\left(\vec{x}, \vec{e}_{\alpha}, t\right)$ depend s on the local macroscopic variables (the water depth $h$ and the velocity $v$ ) and should satisfy the following constraints: 


$$
\sum_{\alpha} f_{\alpha}^{e q}\left(\vec{x}, \vec{e}_{\alpha}, t\right)=h, \quad \sum_{\alpha} \vec{e}_{\alpha} f_{\alpha}^{e q}\left(\vec{x}, \vec{e}_{\alpha}, t\right)=h \cdot v
$$

$f_{\varepsilon}^{n e q}\left(\vec{x}, \vec{e}_{\alpha}, t\right)=f_{\alpha}^{(1)}\left(\vec{x}, \vec{e}_{\alpha}, t\right)+\varepsilon f_{\alpha}^{(2)}\left(\vec{x}, \vec{e}_{\alpha}, t\right)+O\left(\varepsilon^{2}\right)$ is the nonequilibrium distribution function, which has the following cons traints:

$$
\sum_{\alpha} f_{\alpha}^{(k)}\left(\vec{x}, \vec{e}_{\alpha}, t\right)=0, \quad \sum_{\alpha} \vec{e}_{\alpha} f_{\alpha}^{(k)}\left(\vec{x}, \vec{e}_{\alpha}, t\right)=0
$$

For both $k=1$ and $k=2$.

The second-order Taylor expansion is used on (5), it is

$$
\begin{aligned}
\varepsilon\left(\frac{\partial}{\partial t}+\vec{e}_{\alpha} \frac{\partial}{\partial x}\right) f_{\alpha}\left(\vec{x}, \vec{e}_{\alpha}, t\right)+ & \frac{\varepsilon^{2}}{2}\left(\frac{\partial}{\partial t}+\vec{e}_{\alpha} \frac{\partial}{\partial x}\right)^{2} f_{\alpha}\left(\vec{x}, \vec{e}_{\alpha}, t\right)= \\
& -\frac{1}{\tau}\left(f_{\alpha}\left(\vec{x}, \vec{e}_{\alpha}, t\right)-f_{\alpha}^{e q}\left(\vec{x}, \vec{e}_{\alpha}, t\right)\right)+\varepsilon^{2} \cdot g_{\alpha}\left(\vec{x}, \vec{e}_{\alpha}, t\right)
\end{aligned}
$$

Based on (9) and (10), multi-scale expansion is used to each type of (13), and the discrete Lattice Boltzmann equations of different magnitude order are obtained:

$$
\begin{aligned}
& o\left(\varepsilon^{0}\right): \quad f_{\alpha}^{(0)}\left(\vec{x}, \vec{e}_{\alpha}, t\right)=f_{\alpha}^{e q}\left(\vec{x}, \vec{e}_{\alpha}, t\right) \\
& o\left(\varepsilon^{1}\right): \quad f_{\alpha}^{(1)}\left(\vec{x}, \vec{e}_{\alpha}, t\right)=-\tau\left(\frac{\partial}{\partial t_{0}}+\vec{e}_{\alpha} \frac{\partial}{\partial x}\right) f_{\alpha}^{(0)}\left(\vec{x}, \vec{e}_{\alpha}, t\right) \\
& o\left(\varepsilon^{2}\right): \quad-\frac{1}{\tau} f_{\alpha}^{(2)}\left(\vec{x}, \vec{e}_{\alpha}, t\right)+g_{\alpha}\left(\vec{x}, \vec{e}_{\alpha}, t\right)=\frac{\partial f_{\alpha}^{(0)}\left(\vec{x}, \vec{e}_{\alpha}, t\right)}{\partial t_{1}} \\
& +\left(\frac{1}{2}-\tau\right)\left(\frac{\partial}{\partial t_{0}}+\vec{e}_{\alpha} \frac{\partial}{\partial x}\right)^{2} f_{\alpha}^{(0)}\left(\vec{x}, \vec{e}_{\alpha}, t\right)
\end{aligned}
$$

Where

$$
g_{\alpha}\left(\vec{x}, \vec{e}_{\alpha}, t\right)=\frac{i_{e}(x, t)}{3 \varepsilon} \quad \alpha=0,1,2
$$

Zero-order moment of $f_{\alpha}^{e q}\left(\vec{x}, \vec{e}_{\alpha}, t\right)$ is solved which in (14) to (16), and then the macroscopic manifestation can be set:

$$
\begin{aligned}
& \sum_{\alpha} f_{\alpha}^{(0)}=h \\
& \sum_{\alpha} \vec{e}_{\alpha} f_{\alpha}^{(0)}=\beta h^{m} \\
& \sum_{\alpha} \vec{e}_{\alpha}{ }^{2} f_{\alpha}^{(0)}=\frac{m^{2} \beta^{2}}{2 m-1} h^{2 m-1}
\end{aligned}
$$

Where $\beta=\frac{1}{n} \cdot S_{0}^{1 / 2}, m=5 / 3$.

In order to recover the kinematic wave equation, scale adhesion is needed (that is the time scale $t_{0}, t_{1}, t_{2}$ should be restored back to the time scale $t$ ), so let $(15)+\varepsilon \times(16)$ and sum the three directions, thus:

$$
\frac{\partial h}{\partial t}+\frac{\partial\left(\beta h^{m}\right)}{\partial x}=i_{e}(x, t)+o\left(\varepsilon^{2}\right)
$$


Compare to (2), only have the difference of $o\left(\varepsilon^{2}\right)$, where $o\left(\varepsilon^{2}\right)$ can element by adjusting $\tau$. By using the D1Q3 three-speed model, the equilibrium distribution function can be obtained by (18)

$$
\left\{\begin{array}{c}
f_{1}=\frac{1}{2 C^{2}}\left(\frac{m^{2} \beta^{2}}{2 m-1} h^{2 m-1}-C \beta h^{m}\right) \\
f_{0}=\frac{1}{C^{2}}\left(h C^{2}-\frac{m^{2} \beta^{2}}{2 m-1} h^{2 m-1}\right) \\
f_{2}=\frac{1}{2 C^{2}}\left(\frac{m^{2} \beta^{2}}{2 m-1} h^{2 m-1}+C \beta h^{m}\right) \\
g_{1}=g_{0}=g_{2}=\frac{i_{e}(x, t)}{3 \varepsilon}
\end{array}\right.
$$

The initial moment of the distribution function on each node can be instead by the equilibrium distribution function which is calculated by (20). Through the collision step and streaming step of particles, updating the distribution function of each node. According to (11), the depth and velocity on each node can be got.

\section{Application}

In this paper, the numerical simulation of one-dimensional overland flow by Lattice Boltzmann method is compared to analytical solution which furnished by RUI Xiaofang. The depth and unit width discharge on slope bottom are compared respectively. The distributions of depth and unit width discharge along slope when the time equal to 5 minutes and 10 minutes are also discussed. The compared results are shown in Fig.3 to Fig.6.

Good fitting results are shown in these comparison figures. The results of them which are calculated by Lattice Boltzmann method are greatly similar to the results of analytical solution. Before equilibrium time, there are differences of the equilibrium position along slope between Lattice Boltzmann method and analytical solution. The equilibrium position which are calculated by Lattice Boltzmann method is farther than analytical solution. It has something to do with the relaxation time $\tau$.

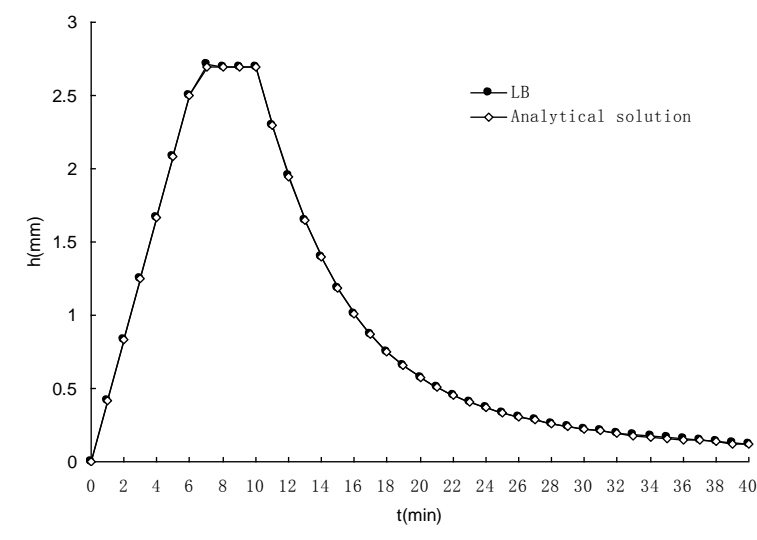

Figure 3. The depth

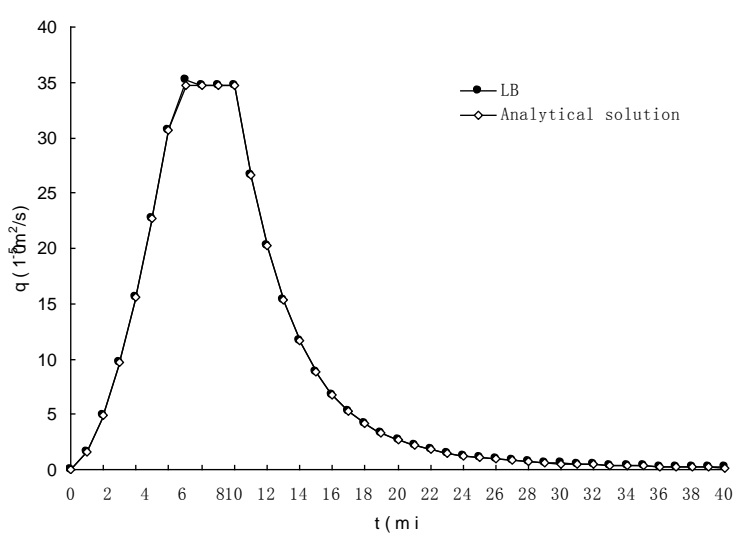

Figure 4. The unit width discharge 

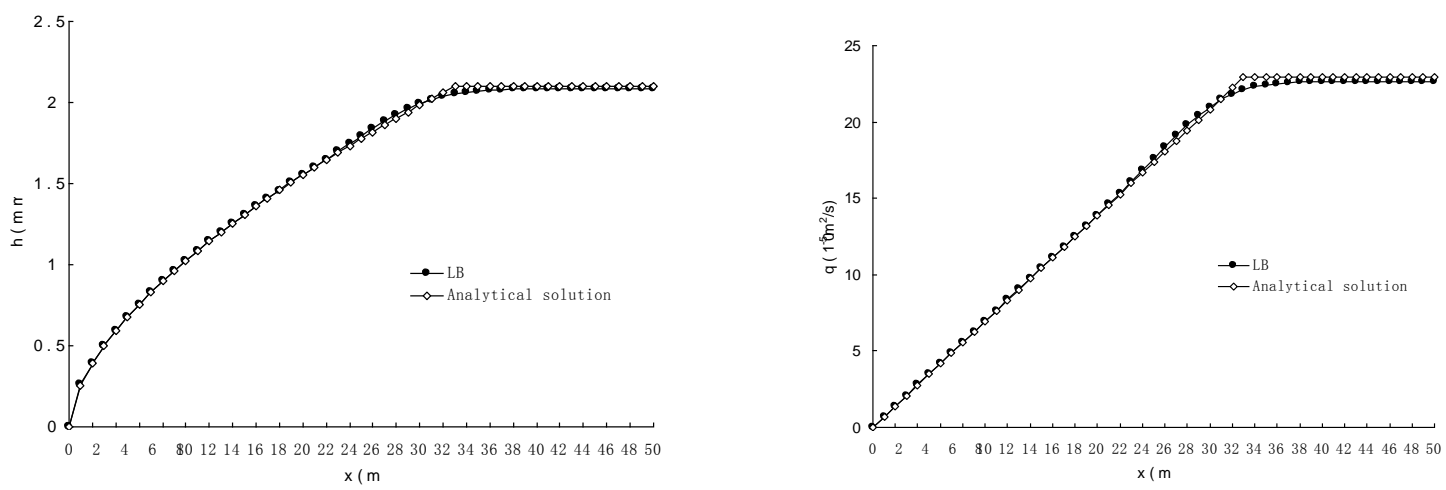

Figure 5. The distributions of depth and unit width discharge along slope when the time equal to 5 (min)
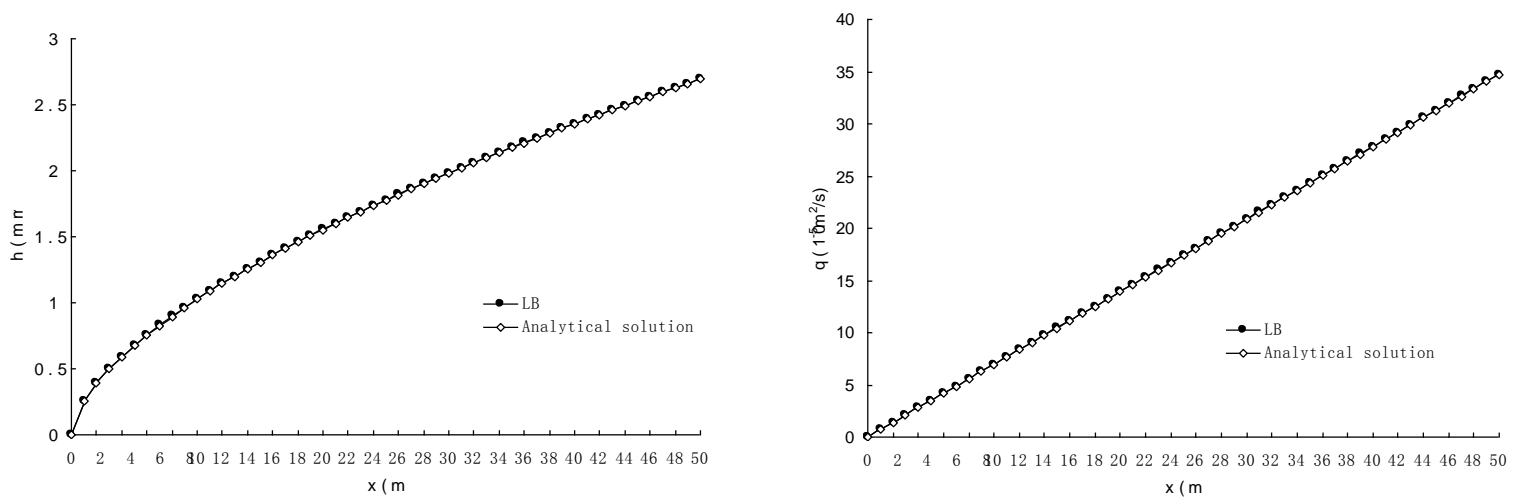

Figure 6. The distributions of depth and unit width discharge along slope when the time equal to 10 (min)

In theory, in order to meet numerical stability, the relaxation time $\tau$ should be greater than 0.5 . However, from the view of calculation accuracy, in order to get a minimum error, an optimal range of $\tau$ should be determined. Equilibrium time $\left(t_{e}\right)$ and the relative error of maximum flow on slope bottom $\left(E_{r}\right)$ are selected to be standards. The result of Lattice Boltzmann method will be compared to analytical solution, and then the optimal range of $\tau$ is discussed. Because relaxation time $\tau$ has something to do with the selection of space step (dx) and time step (dt), four different methods of step selection are chose. They are: (1) $d x=1(\mathrm{~m}), \mathrm{dt}=1(\mathrm{~s})$ (2) $\mathrm{dx}=2(\mathrm{~m}), \mathrm{dt}=1(\mathrm{~s})$ (3) $\mathrm{dx}=1(\mathrm{~m}), \mathrm{dt}=0.1(\mathrm{~s})$ (4) $\mathrm{dx}=2(\mathrm{~m}), \mathrm{dt}=0.1(\mathrm{~s})$. Comparison results are shown in Table 1.

Table 1. Comparison results of $t_{e}$ and $E_{r}$ between LBM and analytical solution

\begin{tabular}{|c|c|c|c|c|c|c|c|c|c|c|c|}
\hline \multirow{3}{*}{$\begin{array}{c}\text { Step } \\
\text { selection }\end{array}$} & \multicolumn{10}{|c|}{ relaxation time $\tau$} & \multirow{3}{*}{$\begin{array}{c}\text { Analytical } \\
\text { solution } \\
t_{e}[\mathrm{~s}]\end{array}$} \\
\hline & \multicolumn{2}{|c|}{0.8} & \multicolumn{2}{|c|}{0.9} & \multicolumn{2}{|c|}{1.2} & \multicolumn{2}{|c|}{1.5} & \multicolumn{2}{|c|}{2.0} & \\
\hline & $t_{e}[\mathrm{~s}]$ & $E_{r}[\%]$ & $t_{e}[\mathrm{~s}]$ & $E_{r}[\%]$ & $t_{e}[\mathrm{~s}]$ & $E_{r}[\%]$ & $t_{e}[\mathrm{~s}]$ & $E_{r}[\%]$ & $t_{e}[\mathrm{~s}]$ & $E_{r}[\%]$ & \\
\hline (1) & - & - & 405 & 1.50 & 420 & 1.50 & 431 & 1.63 & 446 & 1.82 & \\
\hline (2) & 402 & 1.49 & 418 & 1.46 & 444 & 1.58 & 463 & 1.66 & 490 & 1.73 & 388 \\
\hline (3) & 398 & 0.38 & 408 & 0.64 & 425 & 0.84 & 436 & 0.94 & 452 & 1.05 & \\
\hline (4) & 405 & 0.57 & 421 & 0.99 & 448 & 1.20 & 467 & 1.25 & 494 & 1.26 & \\
\hline
\end{tabular}

(Node: "-" indicates numerical instability) 


\section{Conclusion}

The Lattice Boltzmann method which different from conventional numerical methods is used to solve the Kinematic wave equation in this paper. This method provides an indirect way to solve the the partial differential equation. It defines the local equilibrium distribution function $f_{\alpha}^{e q}\left(\vec{x}, \vec{e}_{\alpha}, t\right)$, and gets macroscopic variables by establishing the relationship between $f_{\alpha}^{e q}\left(\vec{x}, \vec{e}_{\alpha}, t\right)$ and macroscopic variables. The depth hydrograph and unit width discharge hydrograph on slope bottom, the distributions of depth and unit width discharge along slope are compared to them of analytical solution. Good fitting results are got. In addition, the selection of relaxation time $\tau$ is discussed. The selection of relaxation time $\tau$ has something to do with the Equilibrium time and the relative error of maximum flow on slope bottom. An optimal range of $\tau$ which from 0.9 to 1.2 is got in this paper. However, the range can be enlarged properly when space step and time step select smaller.

\section{References}

[1] Vieira,J.H.D., "Cond itions governing the use of approximations for the Saint Venant equations for the shallow surface water flow," J.Hydrol., 1983 (60):43-58.

[2] Ponce, V.M., Ruh-Ming Li, Simmons, D.B. "Applicability of kinematic and diffusion models," J. Hydraul. Eng., 1978 (104): 353-360.

[3] Kamahara, M. Yokoyama, T. "Finite element method for direct runoff process," J. Hydraul. Div. Am. Soc. Civ. Eng. 1980 (106) 519-534.

[4] Iwasa, Y. Inouce, k. "Mathematical simulations of channel and overland flows in view of flood disaster engineering," Journal of Natural Disaster Science. 1982 (4)1-30.

[5] Chen S. Y, Doolen G.D., "Lattice Boltzmann method for fluid flows," Annu. Fluid Mech., 1998, (3):314-322.

[6] Luo L-S, "Theory of the Lattice Boltzmann method: Lattice Boltzmann method for no ideal gases," Phys. Rev. E 2000, (62): 4982.

[7] Succi S, Foti E, Higuera FJ, "3-Dimendional flows in complex geometries with the Lattice Boltzmann method,” Europhys. Lett, 1989, (10):433.

[8] Filippova O, Hanel D, "Lattice Boltzmann simulation of gas-particle flow in filtes," Computer \& Fluids, 1997, (26): 697-712.

[9] J.G. Zhou, "A Lattice Boltzmann model for the shallow water equations," Comput. Method Appl. Wech Engrg. 2002 (191):3527-3539.

[10] J. Zhang, G Yan, "Lattice Boltzmann method for one and two dimensional Burgers equation," Physica A 2008, doi: 10.1016/j.physa.2008.04.002.

[11] Xiaofang Rui, "The theory of runoff and concentration," Beijing: China water conservancy and hydropower publishing house, 1995.

[12] P.L.Bhatnagar, E.P. Gross, M. Krook, "A model for collision processes in gases. I .Small amplitude processes in charged and neutral one-component system," Phys.Rev. 1954 (94):511-525. 